\title{
Analysis and Controller Design of Discrete-time Linear Systems with State Saturation
}

\author{
Wei Guan and Guang-Hong Yang
}

\begin{abstract}
This paper studies the problem of stability analysis and controller design for discrete-time linear time invariant (LTI) systems with state saturation. Both full state saturation and partial state saturation are considered. Firstly, a new system model is constructed for solving the key problem. Then, a new method is presented for estimating the domain of attraction of the origin for a system with state saturation. Based on this method, an LMI-based ( linear matrix inequality) iterative algorithms is proposed for determining if a given ellipsoid is contractively invariant. Moreover, an LMI-based algorithm is developed for designing dynamic output-feedback controllers which guarantee that the domain of attraction of the origin for the closed-loop system is as large as possible. An example is given to illustrate the efficiency of the design method.
\end{abstract}

\section{INTRODUCTION}

Control systems with state saturation are often encountered in a variety of applications, including signal processing, recurrent neural networks and control systems, and have been studied extensively (see, e.g., [1]-[7], and the references therein). When there are state saturation in control systems, most of such systems can be modeled by statespace representations with polyhedral or ellipsoidal state constraints, and global stability of an otherwise stable linear closed-loop system can not in general be ensured. A few approaches to the global asymptotic stability of such system were presented in [7]-[10].

For second order systems with state saturation, necessary and sufficient conditions for global asymptotic stability were established in [7] and [9]. For higher order systems, various sufficient conditions for the global asymptotic stability were identified (see, e.g., [6], [10], and the references therein). Under the sufficient condition of [11], any system trajectory starting from inside the state saturation region will never reach the boundary of it, i.e., the state never saturates. This saturation avoidance sufficient condition leads to a degree of conservatism

Extensions of the results of [12] and [13] to the situation involving partial state saturation have been carried out in

This work was supported in part by the Funds for Creative Research Groups of China (No. 60521003), the State Key Program of National Natural Science of China (Grant No. 60534010), National 973 Program of China (Grant No. 2009CB320604), the Funds of National Science of China (Grant No. 60674021), the 111 Project (B08015) and the Funds of PhD program of MOE, China (Grant No. 20060145019).

Wei Guan is with the Department of Automation, Shenyang Institute of Aeronautical Engineering, Shenyang 110136, China; he is also with the College of Information Science and Engineering, Northeastern University, Shenyang, Liaoning, 110004, China. guanweihaha@163.com

Guang-Hong Yang is with the College of Information Science and Engineering, Northeastern University, Shenyang, Liaoning, 110004, China. yangguanghong@ise.neu.edu.cn
[14] and [15], respectively. This problem was reconsidered in [16] and a less conservative result was obtained. Using biconvex programming and static output feedback approach, $H_{\infty}$ control problem was also considered [17].

This paper revisits the problems of stability analysis and controller design for discrete-time LTI systems with state saturation. Both full state saturation and partial state saturation are considered. Firstly, a new system model is constructed for solving the key problem. Then, a method is presented for estimating the domain of attraction of the origin for a system under state saturation. Based on this method, an LMI-based iterative algorithms is proposed for determining if a given ellipsoid is contractively invariant. Moreover, an LMI-based algorithm is developed for designing dynamic output-feedback controllers which guarantee that the domain of attraction of the origin for the closed-loop system is as large as possible.

The paper is organized as follows. Problem statement is given in Section 2. A condition for set invariance is presented for discrete-time LTI systems with state saturation in Section 3. In Section 4 the estimation of domain of attraction is presented. In Section 5 A controller design method based on LMIs is given. An example is presented in Section 6 to demonstrate the proposed design method. Finally, the paper will be concluded in Section 7.

\section{PROBLEM STATEMENT AND PRELIMINARIES}

In this paper, we will consider two classes of discretetime linear systems under state constraints. The first class of systems are defined as

$$
\begin{aligned}
x_{p}(k+1) & =\sigma\left[A_{p} x_{p}(k)+B_{p} u\right] \\
y(k) & =C_{p} x_{p}(k)
\end{aligned}
$$

where $x_{p}(k) \in R^{n_{p}}$ is the plant state, $u \in R^{m}$ is the control input, $y \in R^{p}$ is the measured output. $A_{p}, B_{p}, C_{p}$ are known constant matrices of appropriate dimensions.

The state nonlinearity with the consideration of a piecewise-linear saturation is described as

$$
\sigma\left(x_{i}\right)=\left\{\begin{array}{lr}
x_{i}, & \left|x_{i}\right| \leq x_{i}^{\max }, \\
\operatorname{sign}\left(x_{i}\right) x_{i}^{\max }, & \left|x_{i}\right|>x_{i}^{\max },
\end{array}\right.
$$

for $i \in \mathbf{I}\left[1, n_{p}\right]$. Here we have slightly abused the notation by using $\sigma$ to denote both the scalar valued and the vector valued saturation functions.

System (1) is defined on a closed hypercube as all state variables are constrained to the hypercube. For this reason, system (1) is sometimes called as discrete-time LTI systems 
under state saturation, that is, saturation occurs in the state $x_{i}$ if $\left|x_{i}\right|>x_{i}^{\max }$.

The other class of systems are discrete-time linear systems with partial state saturation and are formulated as

$$
\begin{aligned}
x_{p 1}(k+1) & =\sigma\left[A_{p 11} x_{p 1}(k)+A_{p 12} x_{p 2}(k)+B_{p 1} u(k)\right] \\
x_{p 2}(k+1) & =A_{p 21} x_{p 1}(k)+A_{p 22} x_{p 2}(k)+B_{p 2} u(k) \\
y(k) & =C_{p 1} x_{p 1}(k)+C_{p 2} x_{p 2}(k)
\end{aligned}
$$

where $x_{p 1}(k) \in R^{n_{p 1}}$ is the plant state with sate saturation, $x_{p 2}(k) \in R^{n_{p}-n_{p 1}}$ is the plant state without state saturation, $u \in R^{m}$ is the control input, $y \in R^{p}$ is the measured output. $A_{p 11}, A_{p 12}, A_{p 21}, A_{p 22}, B_{p 1}, B_{p 2}, C_{p 1}, C_{p 2}$ are known constant matrices of appropriate dimensions. Notes that all the state variables are under state saturation if $n_{p 1}=n_{p}$, in which case, system (3) reduces to system (1).

The following definitions and lemma will be used in the sequel.

Definition 1: For a vector $x$, define

$$
\wp\left(n, n_{p}\right)=\left\{x \in R^{n}:\left|x_{i}\right| \leq x_{i}^{\max }, i \in \mathbf{I}\left[1, n_{p}\right], \quad n \geq n_{p}\right\},
$$

then $\wp\left(n, n_{p}\right)$ is the state saturation region.

Definition 2: For a matrix $M \in R^{n_{p 1} \times\left(n_{p}+n_{c}\right)}$, denote the $i$ th row of $M$ as $M_{i}$, define

$$
\mathfrak{I}(M)=\left\{\xi \in R^{n_{p}+n_{c}}: \quad\left|M_{i} \xi\right| \leq x_{i}^{\max }, \quad i \in \mathbf{I}\left[1, n_{p 1}\right]\right\},
$$

For $x(0)=x_{0} \in R^{n}$, denote the state trajectory of system (1) as $\psi\left(k, x_{0}\right)$. Then the domain of attraction of the origin is

$$
\ell:=\left\{x_{0} \in R^{n}: \quad \lim _{k \rightarrow \infty} \psi\left(k, x_{0}\right)=0\right\} .
$$

Consider the following two systems

$$
\begin{aligned}
& \left\{\begin{array}{l}
x_{a 1}(k+1)=\sigma\left[f_{1}\left(x_{a 1}(k), x_{a 2}(k)\right)+g_{1}\left(u_{a}(k)\right)\right], \\
x_{a 2}(k+1)=f_{2}\left(x_{a 1}(k), x_{a 2}(k)\right)+g_{2}\left(u_{a}(k)\right), \\
y_{a}(k)=C_{p 1} x_{a 1}(k)+C_{p 2} x_{a 2}(k), \\
x_{c a}(k+1)=f_{3}\left(x_{c a}(k)\right)+g_{3}\left(y_{a}(k)\right), \\
u_{a}(k)=f_{4}\left(x_{c a}(k)\right)+g_{4}\left(y_{a}(k)\right),
\end{array}\right. \\
& \left\{\begin{array}{l}
x_{\mathrm{b} 1}(k+1)=f_{1}\left(\sigma\left[x_{b 1}(k)\right], x_{b 2}(k)\right)+g_{1}\left(u_{b}(k)\right), \\
x_{b 2}(k+1)=f_{2}\left(\sigma\left[x_{b 1}(k)\right], x_{b 2}(k)\right)+g_{2}\left(u_{b}(k)\right), \\
y_{b}(k)=C_{p 1} \sigma\left[x_{b 1}(k)\right]+C_{p 2} x_{b 2}(k), \\
x_{c b}(k+1)=f_{3}\left(x_{c b}(k)\right)+g_{3}\left(y_{b}(k)\right), \\
u_{b}(k)=f_{4}\left(x_{c b}(k)\right)+g_{4}\left(y_{b}(k)\right),
\end{array}\right.
\end{aligned}
$$

where, $f_{i}(\cdot)$ and $g_{j}(\cdot), i \in \mathbf{I}[1,4], j \in \mathbf{I}[1,2]$ are linear functions. Denote the state trajectory of system (4) as $\psi_{a}\left(t, x_{a}(0)\right)$, and denote the state trajectory of system (5) as $\psi_{b}\left(t, x_{b}(0)\right)$. Let $x_{a}(k)=\left[\begin{array}{l}x_{a 1}(k) \\ x_{a 2}(k) \\ x_{c a}(k)\end{array}\right], x_{b}(k)=\left[\begin{array}{l}x_{b 1}(k) \\ x_{b 2}(k) \\ x_{c b}(k)\end{array}\right]$, then the following lemma is given

Lemma 1: For any initial state $x_{a}(0)=x_{b}(0) \in \wp\left(n, n_{p 1}\right)$, the following two statements are equivalent.

(I) $\lim _{k \rightarrow \infty} \psi_{a}\left(k, x_{a}(0)\right)=0$

(II) $\lim _{k \rightarrow \infty} \psi_{b}\left(k, x_{b}(0)\right)=0$

Proof:

$$
x_{a}(0)=x_{b}(0) \in \wp\left(n, n_{p 1}\right) \Rightarrow\left\{\begin{array}{c}
x_{a 1}(0)=\sigma\left[x_{b 1}(0)\right] \\
x_{a 2}(0)=x_{b 2}(0) \\
x_{c a}(0)=x_{c b}(0)
\end{array}\right.
$$

For $k=0,1,2, \ldots, \infty$,

$$
\begin{aligned}
& \left\{\begin{array}{c}
x_{a 1}(k)=\sigma\left[x_{b 1}(k)\right] \\
x_{a 2}(k)=x_{b 2}(k) \\
x_{c a}(k)=x_{c b}(k)
\end{array}\right. \\
& \Rightarrow\left\{\begin{array}{c}
f_{1}\left(x_{a 1}(k), x_{a 2}(k)\right)=f_{1}\left(\sigma\left[x_{b 1}(k)\right], x_{b 2}(k)\right) \\
f_{2}\left(x_{a 1}(k), x_{a 2}(k)\right)=f_{2}\left(\sigma\left[x_{b 1}(k)\right], x_{b 2}(k)\right) \\
y_{a}(k)=y_{b}(k) \\
u_{a}(k)=u_{b}(k)
\end{array}\right. \\
& \Rightarrow\left\{\begin{array}{c}
f_{1}\left(x_{a 1}(k), x_{a 2}(k)\right)+g_{1}\left(u_{a}(k)\right) \\
=f_{1}\left(\sigma\left[x_{b 1}(k)\right], x_{b 2}(k)\right)+g_{1}\left(u_{b}(k)\right) \\
f_{2}\left(x_{a 1}(k), x_{a 2}(k)\right)+g_{2}\left(u_{a}(k)\right) \\
=f_{2}\left(\sigma\left[x_{b 1}(k)\right], x_{b 2}(k)\right)+g_{2}\left(u_{b}(k)\right) \\
x_{c a}(k+1)=x_{c b}(k+1)
\end{array}\right. \\
& \Rightarrow\left\{\begin{array}{c}
x_{a 1}(k+1)=\sigma\left[x_{b 1}(k+1)\right] \\
x_{a 2}(k+1)=x_{b 2}(k+1) \\
x_{c a}(k+1)=x_{c b}(k+1)
\end{array}\right.
\end{aligned}
$$

In addition, we have

$$
\lim _{k \rightarrow \infty} x_{b 1}(k)=0 \Rightarrow \lim _{k \rightarrow \infty} \sigma\left(x_{b 1}(k)\right)=0 \Rightarrow \lim _{k \rightarrow \infty} x_{a 1}(k)=0
$$

and

$$
\lim _{k \rightarrow \infty} x_{a 1}(k)=0 \Rightarrow \lim _{k \rightarrow \infty} \sigma\left(x_{b 1}(k)\right)=0 \Rightarrow \lim _{k \rightarrow \infty} x_{b 1}(k)=0 .
$$

This completes the proof.

Problem 1: The design problem under consideration is to find a controller such that, the domain of asymptotic stability is enlarged as possible for closed-loop system with state saturation.

\section{A CONDITION FOR SET INVARIANCE}

In this section, we will establish new sufficient conditions for global asymptotic stability for both systems (1) and (3). Because all the state variables are under state saturation if $n_{p 1}=n_{p}$, in which case, system (3) reduces to system (1), we need only to consider system (3). To this end, we first establish a new system as follows.

$$
\begin{aligned}
x_{p 1}(k+1) & =A_{p 11} \sigma\left[x_{p 1}(k)\right]+A_{p 12} x_{p 2}(k)+B_{p 1} u(k) \\
x_{p 2}(k+1) & =A_{p 21} \sigma\left[x_{p 1}(k)\right]+A_{p 22} x_{p 2}(k)+B_{p 2} u(k) \\
y(k) & =C_{p 1} \sigma\left[x_{p 1}(k)\right]+C_{p 2} x_{p 2}(k)
\end{aligned}
$$

which can be rewritten as

$$
\begin{aligned}
x_{p}(k+1) & =A_{p}\left[\begin{array}{c}
\sigma\left(x_{p 1}(k)\right) \\
x_{p 2}(k)
\end{array}\right]+B_{p} u(k) \\
y(k) & =C_{p}\left[\begin{array}{c}
\sigma\left(x_{p 1}(k)\right) \\
x_{p 2}(k)
\end{array}\right]
\end{aligned}
$$

where

$$
\begin{aligned}
A_{p} & =\left[\begin{array}{ll}
A_{p 11} & A_{p 12} \\
A_{p 21} & A_{p 22}
\end{array}\right], B_{p}=\left[\begin{array}{l}
B_{p 1} \\
B_{p 2}
\end{array}\right], \\
C_{p} & =\left[\begin{array}{ll}
C_{p 1} & C_{p 2}
\end{array}\right],
\end{aligned}
$$


and $x_{p}(k) \in R^{n_{p}}$ is the plant state, $x_{p 1}(k) \in R^{n_{p 1}}$ is the plant state with sate saturation, $x_{p 2}(k) \in R^{n_{p}-n_{p 1}}$ is the plant state without state saturation, $u \in R^{m}$ is the control input, $y \in R^{p}$ is the measured output. $A_{p}, B_{p}, C_{p}$ are known constant matrices of appropriate dimensions.

To formulate the corresponding LMIs, we need to introduce additional notation which corresponds to representing the closed-loop system in a compact way. Then, the following equation is given

$$
q(k)=x_{p 1}(k)-\sigma\left(x_{p 1}(k)\right)
$$

Then, we have

$$
\begin{aligned}
x_{p}(k+1) & =A_{p} x_{p}(k)-A_{p 1} q(k)+B_{p} u(k) \\
y(k) & =C_{p} x_{p}(k)-C_{p 1} q(k)
\end{aligned}
$$

where $A_{p 1}=\left[\begin{array}{l}A_{p 11} \\ A_{p 21}\end{array}\right]$.

The controller structure is chosen as

$$
\begin{array}{r}
x_{c}(k+1)=A_{k} x_{c}(k)+B_{k} y(k) \\
u(k)=C_{k} x_{c}(k)+D_{k} y(k)
\end{array}
$$

Next, define the overall state variable $x \in \mathbf{R}^{n}$, where $n=$ $n_{p}+n_{c}$, as

$$
x=\left[\begin{array}{ll}
x_{p}^{T} & x_{c}^{T}
\end{array}\right]^{T}
$$

which allows the linear dynamics of the plant and controller to be combined and written as

$$
x(k+1)=A_{e} x(k)+B_{e} q(k)
$$

where

$$
\begin{aligned}
A_{e} & =\left[\begin{array}{cc}
A_{p}+B_{p} D_{k} C_{p} & B_{p} C_{k} \\
B_{k} C_{p} & A_{k}
\end{array}\right] \\
B_{e} & =\left[\begin{array}{c}
-A_{p 1}-B_{p} D_{k} C_{p 1} \\
-B_{k} C_{p 1}
\end{array}\right]
\end{aligned}
$$

Remark 1: When full state saturation is considered, system (9) can be replaced by the following system

$$
x(k+1)=A_{e} x(k)+B_{e} q(k)
$$

where

$$
\begin{aligned}
A_{e} & =\left[\begin{array}{cc}
A_{p}+B_{p} D_{k} C_{p} & B_{p} C_{k} \\
B_{k} C_{p} & A_{k}
\end{array}\right] \\
B_{e} & =\left[\begin{array}{c}
-A_{p}-B_{p} D_{k} C_{p} \\
-B_{k} C_{p}
\end{array}\right]
\end{aligned}
$$

Definition 4: Let $P \in R^{n \times n}$ be a positive-define matrix. Denote

$$
\varepsilon(P, \delta)=\left\{x \in R^{n}: \quad x^{T} P x \leq \delta\right\} .
$$

Assume that the standard dynamic output feedback controller has been designed. Then, for system (6) controlled by designed controller (8), the following lemma is presented to estimate the domain of attraction of the origin.
Lemma 2: For system (6) given a ellipsoid $\varepsilon(P, 1), P \in$ $R^{n \times n}$, if there exist matrices $Q>0, U>0, G$ such that

$$
\left[\begin{array}{ccc}
-Q & \left(Q\left[\begin{array}{l}
I \\
0
\end{array}\right]-G^{T}\right) & Q A_{e}^{T} \\
* & -2 U & U B_{e}^{T} \\
* & * & -Q
\end{array}\right]<0
$$

for $\varepsilon(P, 1) \subset \mathfrak{I}(M)$, i.e., $\left|M_{i} x\right| \leq x_{i}^{\max }$ for all $x \in \varepsilon(P, 1), i \in$ $\mathbf{I}\left[1, n_{p 1}\right]$, then $\varepsilon(P, 1)$ is a contractively invariant set.

Proof: Choose the following Lyapunov function

$$
V(k)=x(k)^{T} P x(k)
$$

Let $M=G Q^{-1}, W=U^{-1}, P=Q^{-1}$. We have inequality (11) is equivalent to

$$
\begin{aligned}
& {\left[\begin{array}{ccc}
-Q & \left(Q\left[\begin{array}{l}
I \\
0
\end{array}\right]-Q M^{T}\right) & Q A_{e}^{T} \\
* & -2 W^{-1} & W^{-1} B_{e}^{T} \\
* & * & -Q
\end{array}\right]<0} \\
& \Leftrightarrow\left[\begin{array}{cc}
-P & \left(\left[\begin{array}{l}
I \\
0
\end{array}\right]-M^{T}\right) W \\
* & -2 W
\end{array}\right] \\
& +\left[\begin{array}{c}
A_{e}^{T} \\
B_{e}^{T}
\end{array}\right] P P^{-1} P\left[\begin{array}{ll}
A_{e} & B_{e}
\end{array}\right]<0 \\
& \Leftrightarrow\left[\begin{array}{cc}
A_{e}^{T} P A_{e}-P & A_{e}^{T} P B_{e}+\left[\begin{array}{c}
I \\
0
\end{array}\right] W-M^{T} W \\
* & B_{e}^{T} P B_{e}-2 W
\end{array}\right]<0 \\
& \Rightarrow\left[\begin{array}{l}
x \\
q
\end{array}\right]^{T}\left[\begin{array}{c}
A_{e}^{T} P A_{e}-P \\
*
\end{array}\right. \\
& \left.\begin{array}{c}
A_{e}^{T} P B_{e}+\left(\left[\begin{array}{l}
I \\
0
\end{array}\right]-M^{T}\right) W \\
B_{e}^{T} P B_{e}-2 W
\end{array}\right]\left[\begin{array}{l}
x \\
q
\end{array}\right]<0 \\
& \Leftrightarrow V(k+1)-V(k) \\
& +q^{T} W([I 0] x-M x-q)+([I 0] x-M x-q)^{T} W q<0
\end{aligned}
$$

By equation (7) we have that, if $\left|M_{i} x\right| \leq x_{i}^{\max } \quad i \in$ $\mathbf{I}\left[1, n_{p 1}\right]$,

$$
q^{T} W([I 0] x-M x-q)+([I 0] x-M x-q)^{T} W q \geq 0
$$

By employing the $\mathscr{S}$-procedure, it is shown that for any given symmetric positive definite matrix $W$, if

$$
\begin{aligned}
& \left\{A_{e} x+B_{e} q\right\}^{T} P\left\{A_{e} x+B_{e} q\right\}-x^{T} P x \\
& +q^{T} W([I 0] x-M x-q)+([I 0] x-M x-q)^{T} W q<0 \\
& \qquad\left|M_{i} x\right| \leq x_{i}^{\max } \quad i \in \mathbf{I}\left[1, n_{p 1}\right]
\end{aligned}
$$

then

$$
\left\{A_{e} x+B_{e} q\right\}^{T} P\left\{A_{e} x+B_{e} q\right\}-x^{T} P x<0
$$

Thus, the proof is completed. By using Lemma 1 and Lemma 2 , the following theorem is given.

Theorem 1: For the closed-loop system of system (3) and controller (8), given a ellipsoid $\varepsilon(P, 1), P \in R^{n \times n}$, if there exist matrices $Q>0, U>0, G$ such that inequality (11) holds for $\varepsilon(P, 1) \subset \mathfrak{I}(M)$, i.e., $\left|M_{i} x\right| \leq x_{i}^{\max }$ for all $x \in \varepsilon(P, 1), i \in$ 
$\mathbf{I}\left[1, n_{p 1}\right]$, then $\wp\left(n, n_{p 1}\right) \cap \varepsilon(P, 1)$ is a contractively invariant set.

Proof: By Lemma 2, we have that for any initial state $x(0) \in\left(\wp\left(n, n_{p 1}\right) \cap \varepsilon(P, 1)\right)$, the state of system (6) can be attracted to zero. Obviously, $\left(\wp\left(n, n_{p 1}\right) \cap \varepsilon(P, 1)\right) \subset \wp\left(n, n_{p 1}\right)$. Then, by Lemma 1, we have that for any initial state $x(0) \in$ $\left(\wp\left(n, n_{p 1}\right) \cap \varepsilon(P, 1)\right)$, the state of system (3) can be attracted to zero.

\section{ESTIMATION OF THE DOMAIN OF ATTRACTION}

From Theorem 1, we can obtain various sets satisfying the set invariance condition. So, how to choose the largest one of them becomes an interesting problem. Because the set $\wp\left(n, n_{p 1}\right)$ is given, we need only to enlarge the domain $\varepsilon(P, 1)$ as possible for estimating the largest domain of attraction. In this section, we will give a method to find the largest set.

The following definition will be used in the sequel.

Definition 5: Define $X_{R}$ is a prescribed bounded convex set. $X_{R}=\varepsilon(R, 1)=\left\{x \in R^{n \times n}: \quad x^{T} R x \leq 1\right\}, \quad R>0$ or $X_{R}=\operatorname{co}\left\{x_{1}, x_{2}, \ldots, x_{l}\right\}$. For a set $S \in R^{n}, \alpha_{R}(S)=\sup \{\alpha>$ $\left.0: \quad \alpha X_{R} \subset S\right\}$.

With the above shape reference sets, we can choose one from all the $\varepsilon(P, 1)^{\prime} s$ that satisfies the condition of Theorem 1 such that the quantity $\alpha_{R}(\varepsilon(P, 1))$ is maximized. The problem can be formulated as follows

$$
\begin{array}{cl}
\text { sup } & \alpha \\
\text { s.t. } & (a) \\
& (b) \quad \varepsilon(P, 1) \subset \mathfrak{I}(M), \\
& (c) \quad \alpha X_{R} \subset \varepsilon(P, 1)
\end{array}
$$

Condition (b) is equivalent to

$$
\frac{1}{x_{i}^{\max }} M_{i} P^{-1}\left(\frac{1}{x_{i}^{\max }} M_{i}\right)^{T} \leq 1 \Leftrightarrow\left[\begin{array}{cc}
1 & \frac{1}{x_{i}^{\max }} M_{i} P^{-1} \\
* & P^{-1}
\end{array}\right] \geq 0
$$

for all $i \in \mathbf{I}\left[1, n_{p 1}\right]$, where $M_{i}$ be the $j$ th row of $M$.

If the given shape reference set $X_{R}$ is a polyhedron as defined in Definition 5, then Constraint (c) is equivalent to

$$
\left[\begin{array}{cc}
\frac{1}{\alpha^{2}} & x_{j}^{T} \\
* & P^{-1}
\end{array}\right] \geq 0, \quad j \in \mathbf{I}[1, l]
$$

If $X_{R}$ is a ellipsoid $\varepsilon(R, 1)$, then (c) is equivalent to

$$
\frac{R}{\alpha^{2}} \geq P \Leftrightarrow\left[\begin{array}{cc}
\left(1 / \alpha^{2}\right) R & I \\
I & P^{-1}
\end{array}\right] \geq 0 .
$$

If $X_{R}$ is a polyhedron, then from (13), (14), the optimization problem (12) is equivalent to

\section{Algorithm 1:}

$$
\begin{aligned}
& \inf _{Q>0, G} \gamma \\
& \text { s.t. }(a 1)(11) \\
& \quad(b 1)\left[\begin{array}{cc}
1 & \frac{1}{x_{i}^{m a x}} g_{i} \\
* & Q
\end{array}\right] \geq 0, i \in \mathbf{I}\left[1, n_{p 1}\right] \\
& \quad(c 1)\left[\begin{array}{cc}
\gamma & x_{j}^{T} \\
x_{j} & Q
\end{array}\right] \geq 0, j \in[1, l]
\end{aligned}
$$

where $\gamma=1 / \alpha^{2}, Q=\left(\frac{P}{\rho}\right)^{-1}$ and $G=M Q$. Let $g_{i}$ be the $i$ th row of $G$. It is easy to see that all constraints are given in LMIs. If $X_{R}$ is an ellipsoid, we need only to replace (c1) with

$$
\left[\begin{array}{rr}
\gamma R & I \\
I & Q
\end{array}\right] \geq 0 .
$$

\section{Controller Design}

In this section we will design a dynamic output feedback controller (8) such that the estimated domain of attraction is maximized with respect to $X_{R}$.

Lemma 3: For matrix variables $Q>0, U>0, G$ and $K$, constraint (11) is equivalent to constraint (16) as follows

$$
\left[\begin{array}{cccc}
T_{1} & T_{2} & Q A^{T} & Q \bar{C}^{T} \\
* & T_{3} & U B^{T} & U \bar{D}^{T} \\
* & * & T_{4} & \bar{B} K \\
* & * & * & -I
\end{array}\right]<0
$$

where

$$
\begin{aligned}
T_{1} & =-Q-Q \bar{C}^{T} \bar{C} Q_{0}-Q_{0} \bar{C}^{T} \bar{C} Q+Q_{0} \bar{C}^{T} \bar{C} Q_{0} \\
T_{2} & =Q[I 0]^{T}-G^{T}-Q \bar{C}^{T} \bar{D} U_{0}-Q_{0} \bar{C}^{T} \bar{D} U+Q_{0} \bar{C}^{T} \bar{D} U_{0} \\
T_{3} & =-2 U-U \bar{D}^{T} \bar{D} U_{0}-U_{0} \bar{D}^{T} \bar{D} U+U_{0} \bar{D}^{T} \bar{D} U_{0} \\
T_{4} & =-Q-\bar{B} K K_{0}^{T} \bar{B}^{T}-\bar{B} K_{0} K^{T} \bar{B}^{T}+\bar{B} K_{0} K_{0}^{T} \bar{B}^{T} \\
A & =\left[\begin{array}{cc}
A_{p} & 0 \\
0 & 0
\end{array}\right], B=\left[\begin{array}{c}
-A_{p 1} \\
0
\end{array}\right], K=\left[\begin{array}{cc}
A_{k} & B_{k} \\
C_{k} & D_{k}
\end{array}\right], \\
\bar{B} & =\left[\begin{array}{cc}
0 & B_{p} \\
I & 0
\end{array}\right], \bar{C}=\left[\begin{array}{cc}
0 & I \\
C_{p} & 0
\end{array}\right], \bar{D}=\left[\begin{array}{c}
0 \\
-C_{p 1}
\end{array}\right]
\end{aligned}
$$

Proof: Obviously, constraint (11) can be rewritten as follows

$$
T+\left[\begin{array}{ccc}
0 & 0 & \mathrm{Q}(\bar{B} K \bar{C})^{T} \\
* & 0 & U(\bar{B} K \bar{D})^{T} \\
* & * & 0
\end{array}\right]<0
$$

where

$$
T=\left[\begin{array}{ccc}
-Q & Q[I 0]^{T}-G^{T} & Q A^{T} \\
* & -2 U & U B^{T} \\
* & * & -Q
\end{array}\right]
$$

Then, inequality (11) is equivalent to

$$
\begin{aligned}
T & +\left[\begin{array}{c}
0 \\
0 \\
\bar{B} K
\end{array}\right]\left[\begin{array}{lll}
\bar{C} Q & \bar{D} U & 0
\end{array}\right] \\
& +\left(\left[\begin{array}{c}
0 \\
0 \\
\bar{B} K
\end{array}\right]\left[\begin{array}{lll}
\bar{C} Q & \bar{D} U & 0
\end{array}\right]\right)^{T}<0 \\
\Leftrightarrow T & \left.+\left[\begin{array}{c}
0 \\
0 \\
\bar{B} K
\end{array}\right]+\left[\begin{array}{c}
Q \bar{C}^{T} \\
U \bar{D}^{T} \\
0
\end{array}\right]\right)\left(\left[\begin{array}{c}
0 \\
0 \\
\bar{B} K
\end{array}\right]+\left[\begin{array}{c}
Q \bar{C}^{T} \\
U \bar{D}^{T} \\
0
\end{array}\right]\right)^{T} \\
& -\left[\begin{array}{c}
0 \\
0 \\
\bar{B} K
\end{array}\right]\left[\begin{array}{lll}
0 & 0 & K^{T} \bar{B}^{T}
\end{array}\right] \\
& -\left[\begin{array}{c}
Q \bar{C}^{T} \\
U \bar{D}^{T} \\
0
\end{array}\right]\left[\begin{array}{lll}
\bar{C} Q & \bar{D} U & 0
\end{array}\right]
\end{aligned}
$$


In addition, as is known to all that for any matrix $V$, there always exists a matrix $V_{0}$ such that the following inequality holds

$$
\left(V-V_{0}\right)\left(V-V_{0}\right)^{T} \geq 0
$$

Thus, there exist $Q_{0}, K_{0}$ and $U_{0}$ such that (16) is equivalent to (17). On one hand, according to (18), if (17) holds, then (16) holds. On the other hand, when $Q_{0}=Q, K_{0}=K$ and $U_{0}=U,(17)$ holds if (16) holds. Thus, the proof for Lemma 3 is completed.

Remark 2: When full state saturation is considered, only two matrices defined in Lemma 3 should be replaced with

$$
B=\left[\begin{array}{c}
-A_{p} \\
0
\end{array}\right], \bar{D}=\left[\begin{array}{c}
0 \\
-C_{p}
\end{array}\right]
$$

By solving inequalities (11), (b1), (c1), we can solve Problem 1. But constraint (11) is not an LMI, we cannot solve them directly. To overcome this difficulty, we will give the following algorithm by Lemma 3.

Algorithm 2:

Step 1 For system (1), design a standard dynamic output feedback controller such that system (1) is asymptotic stable without considering saturation.

Step 2 Based on the designed controller $K^{*}$ in Step 1, find a feasible set $\left(Q^{*}, U^{*}, G^{*}, \gamma^{*}\right)$ by solving Algorithm 1 . Let $\eta=0$.

Step 3 If $\gamma^{*}-\tilde{\gamma}<\tau$ or $\eta>N$, where $N$ and $\tau>0$ are prescribed numbers, let $\gamma^{*}=\widetilde{\gamma}$ and exit.

Step 4 Let $\gamma^{*}=\widetilde{\gamma}, Q_{0}=Q^{*}, U_{0}=U^{*}, K_{0}=K^{*}$. Solve the following LMI problem

$$
\begin{array}{ll}
\min & \gamma \\
\text { s.t. } & (a 2)(16), \\
& (b 1), \\
& (c 1) \text { or }(c 2),
\end{array}
$$

Step 5 Let $Q^{*}=Q, U^{*}=U, K^{*}=K, \widetilde{\gamma}=\gamma, \eta=\eta+1$. Go to Step 3

\section{EXAMPLES}

Example 1. Consider the system of form (1) with

$$
A=\left[\begin{array}{cc}
-0.5 & 0 \\
-1 & 1.5
\end{array}\right], \quad B=\left[\begin{array}{c}
-0.5 \\
1
\end{array}\right], \quad C=\left[\begin{array}{ll}
1 & 1
\end{array}\right]
$$

and $x_{1}^{\max }=x_{2}^{\max }=1$. Suppose that a controller is given as follows

$$
\begin{aligned}
& A_{k}=\left[\begin{array}{ll}
0.0213 & -1.6320 \\
0.9711 & -1.3363
\end{array}\right], \quad B_{k}=\left[\begin{array}{l}
3.1451 \\
5.0328
\end{array}\right], \\
& C_{k}=\left[\begin{array}{ll}
-0.0040 & -0.2317
\end{array}\right], \quad D_{k}=0
\end{aligned}
$$

Let $X_{R}=\operatorname{co}\left\{\left[\begin{array}{l}1 \\ 1 \\ 0 \\ 0\end{array}\right],\left[\begin{array}{c}1 \\ -1 \\ 0 \\ 0\end{array}\right],\left[\begin{array}{c}-1 \\ 1 \\ 0 \\ 0\end{array}\right],\left[\begin{array}{c}-1 \\ -1 \\ 0 \\ 0\end{array}\right]\right\}$. By using Algorithm 1 we draw the following conclusion

$$
\begin{aligned}
\gamma^{*} & =2.8003 \\
Q^{*} & =\left[\begin{array}{cccc}
2.6171 & 1.0973 & -18.1012 & 1.4457 \\
* & 6.3003 & 0.3067 & 18.5965 \\
* & * & 173.6197 & 28.9220 \\
* & * & * & 93.1208
\end{array}\right] \\
U^{*} & =\left[\begin{array}{ccc}
0.4437 & 0.0708 \\
* & 2.2468
\end{array}\right]
\end{aligned}
$$

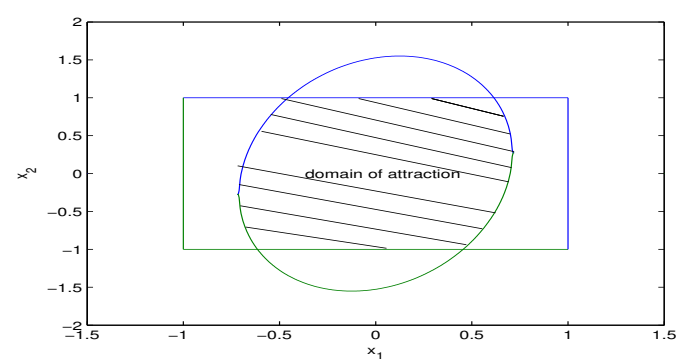

Fig. 1. Cross-section of $\varepsilon\left(P^{*}, 1\right)$ at $x_{c}=0$, under controller (19)

By using Algorithm 2, let $N=3$, we have

$$
\begin{aligned}
\gamma^{*} & =0.8974 \\
Q^{*} & =\left[\begin{array}{cccc}
4.2707 & 1.9588 & -21.6527 & 1.7726 \\
* & 11.1191 & -2.1203 & 20.5999 \\
* & * & 201.6241 & 28.0990 \\
* & * & * & 115.9223
\end{array}\right] \\
U^{*} & =\left[\begin{array}{cc}
1.2363 & -0.8615 \\
* & 1.7134
\end{array}\right] \\
A_{k}^{*} & =\left[\begin{array}{cc}
-0.5342 & -0.1181 \\
0.2839 & 0.0628
\end{array}\right], \quad B_{k}^{*}=\left[\begin{array}{c}
-1.2169 \\
0.6468
\end{array}\right], \\
C_{k}^{*} & =\left[\begin{array}{cc}
-0.1259 & -0.0278
\end{array}\right], \quad D_{k}^{*}=-0.7153
\end{aligned}
$$

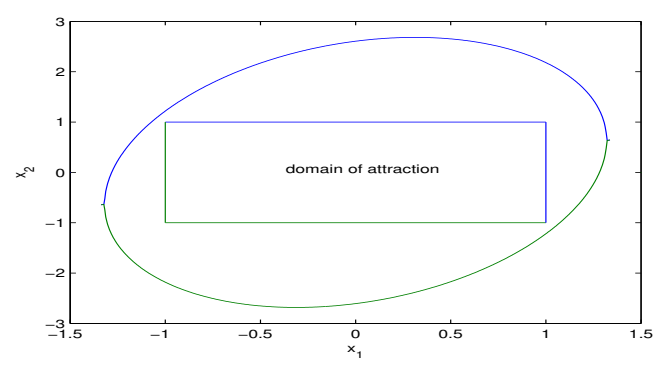

Fig. 2. Cross-section of $\varepsilon\left(P^{*}, 1\right)$ at $x_{c}=0$, under controller (20) 


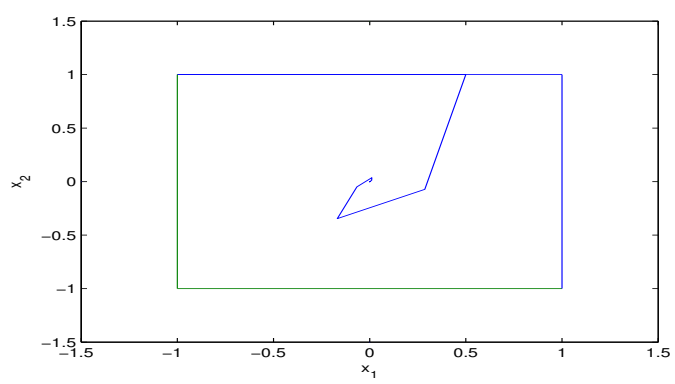

Fig. 3. trajectories of closed-loop systems

\section{CONCLUSIONS}

In this paper, both full state saturation and partial state saturation were considered. A new system was constructed for solving the key problem. Then, LMI-based algorithm was proposed for determining if a given ellipsoid is contractively invariant, and an LMI-based algorithm was developed for constructing dynamic output-feedback controllers which guarantee that the domain of attraction of the origin for the closed-loop system is large as possible. An example was given to illustrate the efficiency of the design method.

\section{REFERENCES}

[1] J. J. Hopfield, Neural computation of decisions in optimization problems, Biol. Cybern, vol. 52, pp. 141-152, 1985.

[2] V. Singh, On global asymptotic stability of 2-D discrete systems with state saturation, Physics Letters A, 372, pp. 5287-5289, 2008.

[3] V. Singh, Improved Criterion for Global Asymptotic Stability of 2D Discrete Systems With State Saturation, IEEE Signal Processing Letters, Vol. 14, pp. 719-722, 2007.

[4] H. Kar and V. Singh, Stability Analysis of 2-D Digital Filters With Saturation Arithmetic: An LMI Approach, IEEE Transactions on Signal Processing, Vol. 53, pp. 2267-2271, 2005.

[5] T. Ooba, On Companion Systems With State Saturation Nonlinearity, IEEE Transactions on Circuits and Systems, Vol. 50, pp. 1580-1584, 2003.

[6] L. Kolev, S. Petrakieva, V. Mladenov, Interval criterion for stability analysis of discrete-time neural networks with partial state saturation nonlinearities, in Proceeding of the 7th Seminar on Neural Network Applications in Electrical Engineering, pp. 11-16, 2004.

[7] L. Hou and A. N. Michel, Asymptotic stability of systems with saturation constraints, in Proc. 35th IEEE Conf. Decision Control, pp. 2624-2629, 1996.

[8] D. Liu and A. N. Michel, Asymptotic stability of systems operating on a closed hypercube, Syst. Control Lett, vol. 19, pp. 281-285, 1992.

[9] R. Mantri, A. Saberi, and V. Venkatasubramanian, Stability analysis of continuous time plannar systems with state saturation nonlinearity, in Proc. IEEE ISCAS96, Atlanta, GA, pp. 60-63, 1996.

[10] H. Fang, L. Lin, Stability analysis for linear systems under state constraints, IEEE Transactions on Automatic Control, vol. 49, pp. 950$955,2005$.

[11] L. Hou and A. N. Michel, Dynamical Systems With Saturation Nonlinearities: Analysis and Design. London, U.K.: Springer-Verlag, 1994.

[12] V. Singh, Elimination of overflow oscillations in fixed-point statespace digital filters using saturation arithmetic, IEEE Transactions on Circuits and Systems, vol. 37, pp. 814-818, 1990.

[13] D. Liu, A. N. Michel, Asymptotic stability of discrete-time systems with with saturation nonlinearities with application to digital filters, IEEE Transactions on Circuits and Systems, vol. 39, pp. 789-807, 1992.

[14] H. Kar, V. Singh, Stability analysis of discrete-time systems in a state-space realization with partial state saturation nonlinearities, IEE Proceedings on Control Theory and Applications, vol. 150, pp. 205208, 2003
[15] D. Liu, A. N. Michel, Stability analysis of systems with partial state saturation nonlinearities, IEEE Transactions on Circuits and Systems, vol. 43, pp. 230-232, 1996.

[16] V. Singh, Stability analysis of discrete-time of discrete-time systems in a state-space realisation with state saturation nonlinearities: linear matrix inequality approach, IEE Proceedings on Control Theory and Applications, vol. 152, pp. 9-12, 2005.

[17] A. T. Neto, E. B. Castelan, and A .Fischman, $H_{\infty}$ control with state constraints, in Proceedings of the 34th Conference on Decision and Control, New Orleans, pp. 2553-2554, December, 1995. 